\title{
Metabolic impact of shift work
}

\author{
Ioná Zalcman Zimberg ${ }^{\mathrm{a}}$, Silvio A. Fernandes Junior ${ }^{\mathrm{b}}$, Cibele Aparecida Crispim ${ }^{\mathrm{c}}$, Sergio Tufik ${ }^{\mathrm{a}}$ and \\ Marco Tulio de Mello ${ }^{\mathrm{a}, \mathrm{b}^{*}}$ \\ ${ }^{a}$ Departamento de Psicobiologia, Universidade Federal de São Paulo, São Paulo, Brazil \\ ${ }^{\mathrm{b}}$ Centro de Estudo Multidisciplinar em Sonolência e Acidentes, São Paulo, Brazil \\ ${ }^{c}$ Faculdade de Medicina, Universidade Federal de Uberlândia, Uberlândia, Minas Gerais, Brazil
}

\begin{abstract}
In developing countries, shift work represents a considerable contingent workforce. Recently, studies have shown that overweight and obesity are more prevalent in shift workers than day workers. In addition, shift work has been associated with a higher propensity for the development of many metabolic disorders, such as insulin resistance, diabetes, dislipidemias and metabolic syndrome. Recent data have pointed that decrease of the sleep time, desynchronization of circadian rhythm and alteration of environmental aspects are the main factors related to such problems. Shortened or disturbed sleep is among the most common health-related effects of shift work. The plausible physiological and biological mechanisms are related to the activation of the autonomic nervous system, inflammation, changes in lipid and glucose metabolism, and related changes in the risk for atherosclerosis, metabolic syndrome, and type II diabetes. The present review will discuss the impact of shift work on obesity and metabolic disorders and how disruption of sleep and circadian misalignment may contribute to these metabolic dysfunctions.
\end{abstract}

Keywords: Shift work, metabolism, obesity, sleep restriction, circadian misalignment

\section{Introduction}

Modern society is moving toward a pattern of working $24 \mathrm{~h}$ a day. Over the last several decades, there has been a rapid increase in the number of shift workers of all ages worldwide, where the proportion of shift workers is estimated to represent $15 \%$ of the entire working population [1]. In Brazil, there are no comparative data for the total population, but research performed in the Sao Paulo region suggests that $11 \%$ of the working population is involved in shift work [2], probably reflecting the economic growth and industrialization of the country.

Evidence from the literature indicates that shift work is associated with several health problems. In particular, over the longer term there is a higher propensity to develop metabolic and nutritional disorders, such as obesity $[3,4]$, altered nutritional metabolism [5-10], insulin resistance [11], diabetes [7,12-16], dyslipidemias [17,18], metabolic syndrome $[6,8,12,19-21]$, and gastrointestinal disorders [22].
With the gradual and constant increase in the number of shift workers worldwide, there is a growing interest in describing the metabolic processes and in understanding the mechanisms involved in disorders commonly observed in this population. The social alterations in the pattern of activity, rest and eating habits, modify the circadian processes of control of innumerable variables.

Human beings are diurnal creatures such that associate the onset of nocturnal sleep as a transition from catabolic processes of daytime activity to nighttime anabolic processes (e.g. use of energy for growth and repair). However nighttime catabolic processes also occur due to the need for mobilization of endogenous energy during fasting state of sleep. Thus, anabolic and catabolic processes occur in parallel during day and night [23]. With the inversion between day-night activities, the metabolic control in medium to long-term may have negative consequences.

From a chronobiological point of view, the claim that the human species are diurnal, to some extent

\footnotetext{
* Corresponding author: Marco Tulio de Mello. Rua Professor Francisco de Castro, 93. Vila Clementino, São Paulo, SP, Brazil, Zip code 04020-050 Phone/Fax number: + 55115572 0177, E-mail adress: tmello@demello.net.br
} 
explains why night shift workers tend to present, for example, decreased appetite during the night $[24,25]$. From a psychosocial perspective, shift workers commonly experience an incompatibility of their daily routines of meals, which may disrupt the food intake control and eating habits. Health problems tend to somatize as the eating behavior is negatively affected by the change of working hours [26].

Shift work is generally associated with chronic misalignment between the endogenous circadian timing system and the behavioral cycles, including sleep/wake and fasting/feeding cycles [28]. Shift workers often experience symptoms akin to jet lag, with gastrointestinal complaints, fatigue, and sleepiness during the scheduled wake periods, and poor sleep during the daytime sleep attempts [29]. Moreover, chronic circadian misalignment and sleep restriction have been proposed to be the underlying causes for the adverse metabolic effects of shift work [30,31].

This review will discuss the recent findings concerning the impact of shift work on metabolic disorders. It will also be discuss how disruption of sleep and circadian misalignment may contribute to these metabolic dysfunctions.

\section{Methods}

A literature search was conducted in PubMed and Thomson Reuters ISI Web of Knowledge $\AA$ for English, Portuguese and Spanish-language publications. No year constraints were used. Key words such as "shift work", "metabolism", "obesity", and "metabolic disorders" were used, either alone or in combination with each other. In addition, we conducted searches in the reference lists of articles, books, and other relevant literature.

\section{Shift work and metabolic disturbances}

Several studies have shown that shift workers are more prone to develop many health problems when compared to day workers $[6,7,9,25,32,33]$. These health problems include obesity, insulin resistance, type 2 diabetes, dyslipidemia, cardiovascular diseases, metabolic syndrome, and gastrointestinal disorders[6,7,9, 13,14,18-21,25,32-37].

The prevalence of overweight and obesity in shift workers are of about 47.2 and $2.8 \%$, respectively ${ }^{38}$. Morikawa et al. [39] in a study with 1529 male adult workers found that shift workers had a body mass index (BMI) significantly higher than day workers.
When comparing 787 day workers with 787 rotating shift workers, Parkes [32] found that the duration of shift work exposure was a highly significant predictor among the shift workers, after controlling for age. The authors concluded that a longer exposure predicts a higher BMI. Job stress and long working hours also seem to contribute to an increase in BMI [40].

Not only obesity but weight gain is more often in shift workers than those workers with the usual work day [4,41]. Geliebter et al. [41] in a retrospective study analyzed the activity levels and eating behaviors of a group of 49 shift workers with 36 day workers. In the course of two years, those working late or night shifts reported greater weight gain than those working during day (4.2 kg versus $0.9 \mathrm{~kg}$, no difference between genders). Authors pointed that this increase was associated with an increase in food intake, a decrease in exercise, more napping, and a consequent reduction in total estimated energy expenditure. However, Lowden et al. [23] stated that, while the majority of studies demonstrated an association between shift work and weight gain, the causal factors underlying this association is still not well established.

It is also important to note that the occurrence of obesity in shift workers is associated with other metabolic problems. Karlsson et al. [6], in a crosssectional survey of 27.485 people in Sweden, found that shift workers besides having a higher prevalence of obesity, presented high triglycerides, and a low concentration of high-density lipid (HDL) cholesterol than day workers. Other studies $[6,17,18,42,43]$ confirmed that the lipid profiles of shift workers are abnormal.

Regarding glucose metabolism, it has been reported that two- and three-shift systems $[44,45]$ cause significant increases in the risk of developing diabetes mellitus. A recent 14-yr cohort study on day ( $\mathrm{n}=$ $4219)$ and alternating shift workers $(n=2885)$ assed the effect of shift work on glycated hemoglobin $\left(\mathrm{HbA}_{1 \mathrm{c}}\right)$ levels - a measure of the average glycemic level and that the increase precedes the onset of diabetes mellitus - according to entry year [45]. The authors found a dose-response relationship between the duration of shift work and the relative increase in $\mathrm{HbA}_{1 \mathrm{c}}$ levels. Subjects engaged in shift work for 11 years or more had an approximately 2 -fold higher OR than subjects who had never done shift work [45].

Nagaya et al. [15] found that all markers of insulin resistance, including hypertension, hyperglycemia, hypertriglyceridemia, and hypo-high density lipoprotein-cholesterolemia, were more common in shift than in day workers in the age group of less than 50 
years. More recently Padilha et al. [11] showed that fixed early morning and night workers present higher scores of HOMA-IR - an index of insulin resistance.

Evidences have showed that both male and female shift workers of various occupations are more likely to develop metabolic syndrome over a 4 to 5 -year period than day-shift controls [8,12].

A study of permanent night workers indicated that even after two years of regular night work (4-6 nights worked per week), glucose and insulin secretion rhythms were only partially adjusted to night work. This suggests that the negative metabolic consequences would be even greater for those working more rapidly rotating shifts [46].

These metabolic changes may be the result of one or more pathways by which shift work may predispose to cardiovascular disease. A number of other factors have also been associated with metabolic syndrome and cardiovascular disease such as behavioural changes e.g. diet, smoking, sedentary; disturbed socio-temporal patterns e.g. stress; misalignment of circadian rhythms; and sleep debt.

\section{Effect of circadian disruption on metabolism}

As evolutionary heritage, the biological (and not cultural) need of human food consumption is influenced primarily by the necessity of consume enough energy to avoid excessive body weight loss and then by the influence of the circadian time of day in this system. The regulation of hunger and sleep undertaken by the hypothalamus is interconnected to control of circadian phenomena and in both cases these processes regulate the rate of body energy, therefore, the metabolism. The endogenous circadian timing system, including the suprachiasmatic nucleus (SCN) in the hypothalamus and peripheral oscillators in vital organs, optimally regulates much of our physiology and behavior across the 24-h day when it is properly aligned with the sleep/wake cycle [47].

Many hormones involved in metabolism, such as insulin, glucagon, adiponectin, cortisol, growth hormone $(\mathrm{GH})$, leptin, and ghrelin, have been shown to exhibit circadian oscillation with peaks and troughs of activity at different times of day [48]. An important aspect of circadian regulation is that these rhythms are expressed in a particular phase relationship to one another [49]. Thus, for example, during night there is an elevation in GH, leptin and glucose levels. Still in night period it is observed a reduced insulin release from pancreatic beta cells duo to the reduced glucose utilization by non-insulin dependent and insulin dependent tissues during slow wave sleep. On the other hand, cortisol levels increase near the end of the sleep period and contribute to enhanced cardiovascular tone and glucose utilization, two physiological processes that contribute to optimal waking behavior [49].

In shift work normally diurnal behavioral and physiological rhythms are voluntarily or experimentally shifted to abnormal phases of the light-dark cycle, resulting in a misalignment among internal behavioral/physiological rhythms as well as a misalignment between internal rhythms and the external day/night cycle [50]. Thus, shift work is generally associated with chronic misalignment between the endogenous circadian timing system and the behavioral cycles, including sleep/wake and fasting/feeding cycles $[27,28]$ and complete adjustment will seldom be achieved, even in employees on a 'permanent' night shift. Even after prolonged duration of time on this shift, only a minority of night workers $(<3 \%)$ will show phase adaptation of their circadian system to the nocturnal activity pattern [51].

The current work on metabolic disorders in shift workers performed in the field only describes the presence of disease and metabolic risk factors (fasting baseline sample). However, the investigation of the precise mechanisms that are associated with these metabolic disorders has been conducted only in laboratory settings which can lead to some limitations when extrapolating the data.

Scheer et al. [47], in a forced desynchrony protocol with 10 subjects, found that short-term circadian misalignment - similar to what occurs chronically with shift work-, results in systematic increases in postprandial glucose, insulin, and mean arterial pressure, systematic decreases in $24 \mathrm{~h}$ leptin, and the complete inversion of the cortisol profile (increased cortisol at the end of the wake episode and beginning of the sleep episode).

Shea et al. [52], in a study with 6 subjects throughout a constant routine protocol, found that alterations in the sleep/wake schedule would lead to an increased $24 \mathrm{~h}$ range in circulating leptin, with lowest leptin upon awakening, which, by influencing food intake and energy balance, could be implicated in the increased prevalence of obesity in the shift-work population.

In studies conducted by our group in a steel industry with 3 groups of shift workers - fixed early morning, night and day shift - with energy intake and activity levels kept as normal as possible over a 24-hour period, it was demonstrated that fixed early morning 
shift workers where more susceptible to alterations on glucose metabolism [11] and hormones involved in the control of food intake and obesity [10]. Interestingly sleep did not seem to influence these results as the early morning group had longer sleep duration then the other groups.

Circadian variability in gastrointestinal activity is also an important feature to be considered in shift work. Gastrointestinal motility, gastric acidity, enzyme activity and nutrient absorption, are circadian periodic as is liver metabolism.

Konturek et al. [22] indicate that the absorption processes vary depending on: (i) time of day (food intake during night period cause a greater reduction in gastric $\mathrm{pH}$ than during the morning), (ii) decrease satiety during night; (iii) increase insulin resistance during night, and (iv) lower gastrointestinal response after the evening meal, due to the different pattern of electrical and hormonal activity of intestinal tract during this period. Therefore the absorption system appears to be less "willing" to deal with food at night. Food uptake at a different or irregular timing can disrupt certain rhythmic functions and lead to a variety of gastrointestinal symptoms and gastric and duodenal ulcers [53].

As light and food are the two main synchronizing factors of central and peripheral oscillators, respectively [54]. Therefore, it was demonstrated in animals that, when feeding time is scheduled to the nocturnal time or when its daily episodes are suppressed by constant snacking or high-fat feeding, some circadian rhythms driven by peripheral oscillators became desynchronized from the rhythms controlled by SCN and entrained by light $[55,56]$. Whether meals scheduled at abnormal circadian times, as occurs during shift work, also leads to such "internal desynchronization"' in humans has yet to be determined.

The data discussed above clearly demonstrate the adverse metabolic effects of circadian misalignment, a condition that is highly prevalent in shift workers. Although these results are in line with epidemiological data associating shift work with increased risk of obesity and diabetes [29], the laboratory conditions do not mimic "real life" and additional field studies therefore are urgently needed to investigate in more detail the adverse mechanisms of real life circadian desynchronization.

\section{Effect of sleep restriction and disorders in metabolism}

Shift work schedules induce chronic sleep debt some reports refer 5 hours of sleep or less [57] - and these workers experience significantly more sleep disorders than day workers [58].

According to The National Sleep Foundation 2005 Sleep in America poll [59], compared to their day shift counterparts, shift workers are more likely to suffer from insomnia as well as excessive daytime sleepiness $(61 \%$ vs. $47 \%$ and $30 \%$ vs. $18 \%$ respectively). In a Brazilian sample of shift workers it was seen a higher prevalence of obstructive sleep apnea than is commonly described in population ( $9 \%$ vs $4 \%$ respectively) [60,61]. A survey of Long Island Rail Road commuters found that more than half of shift workers reported sleep problems, and 70\% reported napping while on the train [62].

Many studies show that the alteration in sleep time can influence various aspects associated with the nutritional and metabolic balance of the body, such as the control of body mass $[63,64]$ and the control of food intake $[64,65]$, glucose metabolism $[66,67]$ and lipid metabolism $[7,42]$.

In laboratory studies of healthy young adults submitted to recurrent partial sleep restriction, marked alterations in metabolism, including decreased glucose tolerance and insulin sensitivity [68] and altered metabolism of food [65], have been demonstrated.

Sleep curtailment is associated with two parallel endocrine modifications that can significantly alter food intake: decreased secretion of the anorexigenic hormone leptin $[64,65,69]$ and increased secretion of the orexigenic hormone ghrelin $[65,64,70,71]$, thus resulting in increased hunger and food intake [65].

Taheri [72] suggested some hypotheses regarding the mechanism that relates sleep restriction and obesity. According to the author, greater awake time, besides promoting hormonal changes capable of increasing caloric intake, may provide more opportunity for food intake and result in greater fatigue, which tends to decrease the level of physical activity and decrease basal metabolic rate. Significant weight gain may, in turn, result in insulin resistance, which is a condition that may promote further adiposity.

Spiegel and colleagues $[65,68]$ also demonstrated that 6 consecutive nights of sleep restriction $(4 \mathrm{~h}$ of sleep per night) was associated with increases in sympathetic nervous system activity, evening cortisol level and GH level and reductions in thyroidstimulating hormone (TSH). Magee et al. [73] ob- 
served that 2 consecutive nights of $5 \mathrm{~h}$ of sleep led to a significant reduction in PYY level and a corresponding decrease in satiety.

Several studies have shown major changes in glucose tolerance under conditions of sleep restriction or deprivation $[65,68,74]$. In a laboratory study, Spiegel et al. [68] analyzed the glucose tolerance (measured by an intravenous bolus of glucose; $300 \mathrm{mg} / \mathrm{kg}$ body mass) in 11 young men after time in bed had been restricted to $4 \mathrm{~h}$ per night for six nights. The authors compared the sleep-debt condition with measurements taken at the end of a sleep-recovery period (fully rested condition) when participants had been allowed $12 \mathrm{~h}$ in bed per night for six nights. They observed that glucose tolerance was lower in the sleep-loss condition than in the fully rested condition.

The results of this and other studies $[64,69,70,75,76]$ suggest that modification of sleep duration can lead to endocrine misalignment that lead to development of obesity and metabolic disorders.

In the nutritional point of view, sleep deprivation seems to increase appetite and the preference for foods containing more energy [64,77]. Spiegel et al. [71] showed that the appetite for energy-rich nutrients with high carbohydrate content, including sweets, salty snacks and starchy foods, increased from 33\% to $45 \%$, although, appetite for fruits, vegetables and high-protein nutrients was less affected. This preference for such foods is very concerning, since besides presenting a hormone pattern that predisposes to an increased energy intake [71] individuals with sleep loss tend to meet this need with foods of low nutritional quality [78-82].

Nedeltcheva et al. [83] in a 14 consecutive days of sleep restriction protocol (5.5h sleep per night) with ad libitum food intake, indicated that sleep restriction led to an increase in calorie consumption that was attributed to snacking particularly during the night when the individual would normally be sleeping. This greater opportunity to eat during evening, a moment of the day where people become much less satisfied by intake and may be more vulnerable to overeating, could promote a higher susceptible to weight gain [84,85].

Individuals who get insufficient sleep are more likely to experience fatigue and excessive daytime sleepiness, which could yield individuals less likely to engage in daytime physical activity and more likely to engage in sedentary behaviors such as television viewing $[29,72,86,87]$. However, the association between sleep restriction and metabolic/ nutritional disorders in shift workers is little explored in literature, and more studies are needed to clarify these relationship.

The metabolic impact of shift work is summarized in Figure 1 according to what have been proposed in the present study.

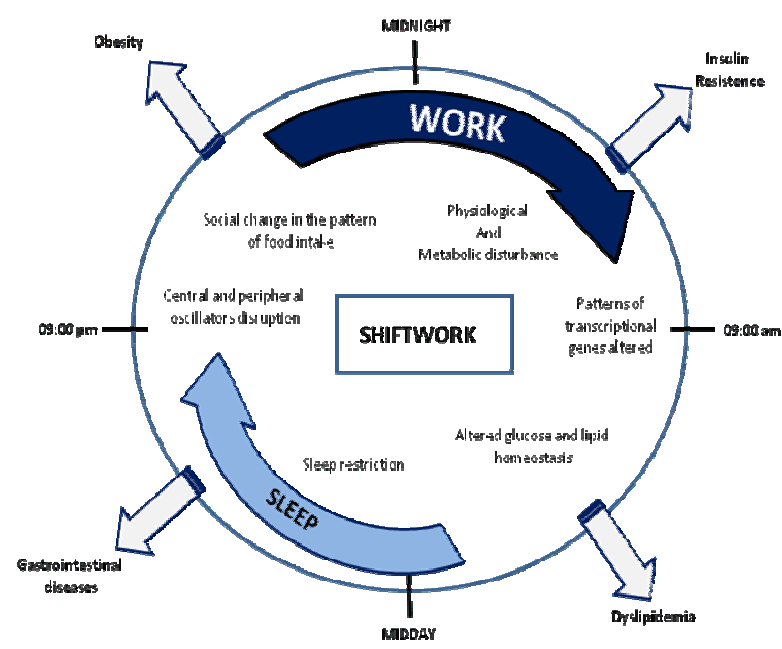

Fig.1. Circadian, physiological and behavioral changes associated with the adverse metabolic effects of shift work

\section{Final considerations}

This review has clearly demonstrated that shift work is associated with an increased occurrence of metabolic disorders, such as insulin resistance, diabetes, dyslipidemias and metabolic syndrome. It is still confounding whether circadian disruption or sleep restriction is the main responsible for the metabolic changes associated with shift work. Recently, pivotal studies have indicated that sleep restriction alone can lead to drastic changes in metabolism [88]. Since some amount of sleep loss usually occurs during circadian disruption, it remains important to determine which aspects of metabolic dysfunction are due to sleep restriction and which are the result of circadian disruption independent of sleep restriction.

Also, there is a variety of shift schedules that differ in their impact on health, depending upon the type of work performed and the exact nature of the work schedules. A better understanding of these differences may make it possible to choose schedules that are less harmful and pose less risk for the worker. 


\section{References}

[1] Bureau of Labor Statistics. Workers on flexible and shift schedules in May 2004. News, U.S. Department of Labor, Bureau of Labor Statistics, Washington, 2005, USDL 05-1198.

[2] F.M. Fischer, O.V. Costa, M.T. Taira and M.I. Watanabe, Shift and day workers: some features of the workers' population, working conditions, accidents. A study of the metropolitan area of São Paulo, Brasil Int. Newslett 12 (1995), 115.

[3] M. Lennernas, T. Akerstedt and L. Hambraeus, Nocturnal eating and serum cholesterol of three-shift workers, Scandinavian Journal of Work and Environmental Health 20 (1994), 401-406.

[4] L.G. van Amelsvoort, E.G. Schouten and F.J. Kok, Duration of shiftwork related to body mass index and waist to hip ratio, Int J Obes Relat Metab Disord 23 (1999), 973-8.

[5] Y. Esquirol, V. Bongard, L. Mabile, B. Jonnier, J.M. Soulat and B. Perret, Shift work and metabolic syndrome: respective impacts of job strain, physical activity, and dietary rhythms, Chronobiol Int 26 (2009), 544-59.

[6] B. Karlsson, A. Knutsson and Lindahl B, Is there an association between shift work and having a metabolic syndrome? Results from a population based study of 27,485 people, Occup Environl Med 58 (2001), 747-752.

[7] B.H. Karlsson, A.K. Knutsson and B.O. Lindahl, Metabolic disturbances in male workers with rotating threeshift work. Results of the WOLF study, Int Arch Occup Environ Health 76 (2003), 424-430.

[8] Y.C. Lin, T.J. Hsiao, P.C. Chen, Persistent shift-work exposure accelerates development of metabolic syndrome among middle-aged female employees: a five-year follow-up, Chronobiol Int 26 (2009), 740-755.

[9] J. Waterhouse, P. Buckley, B. Edwards and T. Reilly, Measurement of, and some reasons for, differences in eating habits between night and day workers, Chronobiol Int 20 (2003), 1075-92.

[10] C.A. Crispim, J. Waterhouse, A.R. Dâmaso , I.Z. Zimberg, H.G. Padilha, L.M. Oyama, S. Tufik and M.T. de Mello, Hormonal appetite control is altered by shift work: a preliminary study, Metabolism 9 (2011). [Epub ahead of print]

[11]H.G. Padilha, C.A. Crispim, I.Z. Zimberg, S. Folkard, S. Tufik and M.T. de Mello, Metabolic responses on the early shift, Chronobiol Int 27 (2010), 1080-92.

[12] A. Pietroiusti, A. Neri, G. Somma, L. Coppeta, I. Iavicoli and A. Bergamaschi, Incidence of metabolic syndrome among night-shift health care workers, Occup Environ Med 67 (2010), $54-7$.

[13]D.J. Gottlieb, N.M. Punjabi, A.B. Newman, H.E. Resnick, S Redline, C.M. Baldwin and F.J. Nieto, Association of sleep time with diabetes mellitus and impaired glucose tolerance, Arch Intern Med 165 (2005),863-867.

[14]P.M. Nilsson, M. Roost, G. Engstrom, B. Hedblad and G. Berglund, Incidence of diabetes in middle-aged men is related to sleep disturbances, Diabetes Care 27 (2004), 2464-2469.

[15] T. Nagaya, H. Yoshida and H. Takahashi, Markers of insulin resistance in day and shift workers aged 30-59 years, Int Arch Occup Environ Health 75 (2002), 562-568.

[16]T. Suwazono, D. Yasushi, M. Oishi, K. Tanaka, E. Kobayashi and K. Sakata, Shiftwork and impaired glucose metabolism: a 14-year cohort study on 7104 male workers, Chronobiol Int 26 (2009), 926-941.

[17] M. Dochi, Y. Suwazono, K. Sakata, Y. Okubo, M. Oishi, K. Tanaka, E. Kobayashi and K. Nogawa, Shift work is a risk factor for increased total cholesterol level: a 14-year prospective cohort study in 6886 male workers, Occup Environ Med 66 (2009), 592-7.

[18] M. Romon, M.C. Nuttens, C. Fievet, P. Pot, J.M. Bard, D. Furon and J.C. Fruchart, Increased triglyceride levels in shift workers, Am J Med 93 (1992), 259-62.

[19] Y. Li, Y. Sato and N. Yamaguchi, Shift work and the risk of metabolic syndrome: a nested case-control study, Int J Occup Environ Health 17 (2011), 154-60.

[20] X.S. Wang, M.E. Armstrong, B.J. Cairns, T.J. Key and R.C. Travis, Shift work and chronic disease: the epidemiological evidence, Occup Med (Lond) 61 (2011), 78-89.

[21]D. Szosland, Shift work and metabolic syndrome, diabetes mellitus and ischaemic heart disease, Int J Occup Med Environ Health 23 (2010), 287-91.

[22]P.C. Konturek, T. Brzozowski and S.J. Konturek, Gut Clock: Implication of circadian rhythms in the gastrointestinal tract, Journal of Physiology and Pharmacology 62 (2011), 139-150.

[23]A. Lowden, C. Moreno, U. Holmback, M. Lennernas and P. Tucker, Eating and shift work - effects on habits, metabolism, and performance Scand, J Work Environ Health 36 (2010), $150-162$.

[24]M. Attia, M.K. Mustafa, M. Khogali, N.A. Mahmoud and E.I. Arar, Optimization of night and shiftwork plans among policemen in Kuwait: a field experiment, Int Arch Occup Environ Health 56 (1985), 81-90.

[25]A. Lowden, U. Holmbäck, T. Åkerstedt, A. Forslund, J. Forslund and M. Lennernäs, Time of day and type of food - relation to mood and hunger during 24 hours of constant conditions, J Hum Ergol (Tokyo) 30 (2001), 381-6.

[26]D. Tepas, Do eating and drinking habits interact with work schedule variables?, Work Stress 4 (1990), 203-11.

[27]R.L. Sack, M.L. Blood and A.J. Lewy, Melatonin rhythms in night shift workers, Sleep 15 (1992), 434-441.

[28] M. Roden, M. Koller, K. Pirich, H. Vierhapper and F. Waldhauser, The circadian melatonin and cortisol secretion pattern in permanent night shift workers, Am J Physiol 265 (1993), 261-267.

[29]A. Knutsson, Health disorders of shift workers, Occup Med (Lond) 53 (2003), 103-108.

[30] R.M. Buijs, F.A. Scheer, F. Kreier, C. Yi, N. Bos, V.D. Goncharuk and A Kalsbeek, Organization of circadian functions: Interaction with the body, Prog Brain Res 153 (2006), 341360 .

[31]A. Kohsaka and J. Bass, A sense of time: How molecular clocks organize metabolism, Trends Endocrinol Metab 18 (2007), 4-11.

[32] K.R. Parkes, Shift work and age as interactive predictors of body mass index among offshore workers, Scand J Work Environ Health 28 (2002), 64-71.

[33]L. Di Lorenzo, G. De Pergola and C. Zocchetti, Effect of shift work on body mass index: results of a study performed in 319 glucose-tolerant men working in a Southern Italian industry, Int J Obes Relat Metab Disord 27 (2003), 1353-1358.

[34]M.A.de Assis, E. Kupek, M.V. Nahas and F. Bellisle, Food intake and circadian rhythms in shift workers with a high workload, Appetite 40 (2003), 175-83.

[35]A. Pietroiusti, A. Neri, G. Somma, L. Coppeta, I. Iavicoli, A Bergamaschi and A. Magrini, Incidence of metabolic syndrome among night-shift healthcare workers, Occup Environ Med 67 (2010), 54-7.

[36] M. Ishizaki, Y. Morikawa and H. Nakagawa, The influence of work characteristics on body mass index and waist to hip ratio in Japanese employees, Ind Health 42 (2004), 41-49.

[37] M. Ha and J. Park, Shiftwork and metabolic risk factors of cardiovascular disease, J Occup Health 47(2005), 89-95. 
[38] L.C. Antunes, R. Levandovski, G. Dantas, W. Caumo and M.P. Hidalgo, Obesity and shift work: chronobiological aspects, Nutr Res Rev 23 (2010), 155-68.

[39] Y. Morikawa, H. Nakagawa, K. Miura, Y. Soyama, M. Ishizaki, T. Kido, Y. Naruse, Y. Suwazono, and K. Nogawa, Effect of shift work on body mass index and metabolic parameters, Scand J Work Environ Health 33 (2007), 45-50.

[40] A.S. Ostry, S. Radi and A.M. Louie, Psychosocial and other working conditions in relation to body mass index in a representative sample of Australian workers, BMC Public Health 6 (2006), 53.

[41] A. Geliebter, M.E. Gluck, M. Tanowitz, N.J. Aronoff and G.K. Zammit, Work-shift period and weight change, Nutrition 16 (2000), 27-9.

[42] M. Ghiasvand, R. Heshmat, R. Golpira, V. Haghpanah, A. Soleimani, P. Shoushtarizadeh, S.M. Tavangar and B. Larijani, Shift working and risk of lipid disorders: a cross-sectional study, Lipids Health Dis 10 (2006), 5- 9.

[43]M. Romon, M.C. Nuttens, C. Fievet, P. Pot, J.M. Bard, D. Furon and J.C. Fruchart, Increased triglyceride levels in shift workers, Am J Med 93(1992), 259-62.

[44] Y. Morikawa, H. Nakagawa and K. Miura, Shift work and the risk of diabetes mellitus among Japanese male factory workers, Scand J Work Environ Health 31 (2005), 179-183.

[45] Y. Suwazono, K. Sakata,Y. Okubo, H. Harada, M. Oishi, E. Kobayashi, M. Uetani, T. Kido and K. Nogawa, Long-term longitudinal study on the relationship between alternating shiftwork and the onset of diabetes mellitus in male Japanese workers, J. Occup. Environ. Med 48 (2006), 455-461.

[46] L. Morgan, S. Hampton and M. Gibbs, Circadian aspects of postprandial metabolism, Chronobiol Int 20 (2003), 795-808.

[47]F.A. Scheer, M.F. Hilton and C.S. Mantzoros, Adverse metabolic and cardiovascular consequences of circadian misalignment, Proc Natl Acad Sci USA 106 (2009), 4453-4458.

[48] O. Froy, The relationship between nutrition and circadian rhythms in mammals, Front Neuroendocrinol 28 (2010), 6171.

[49]A.D. Laposky, J. Bass, A. Kohsaka and F.W. Turek, Sleep and circadian rhythms: key components in the regulation of energy metabolism, FEBS Lett 582 (2008),142-151.

[50]D.M. Arble, K.M. Ramsey, J. Bass and F.W. Turek, Circadian disruption and metabolic disease: findings from animal models, Best Practice \& Research Clinical Endocrinology \& Metabolism 24 (2010), 785-800.

[51]S. Folkard, Do permanent night workers show circadian adjustment? A review based on the endogenous melatonin rhythm, Chronobiol Int 25 (2008), 215-24.

[52] S.A. Shea, M.F.Hilton and C. Orlova, Independent circadian and sleep/wake regulation of adipokines and glucose in humans, J Clin Endocrinol Metab 90 (2005), 2537-2544.

[53] K. Segawa, S. Nakazawa, Y. Tsukamoto, Y. Kurita, H. Goto, A. Fukui and K. Takano, Peptic ulcer is prevalent among shift workers, Dig Dis Sci 32 (1987), 449-53.

[54] M.J. Prasai, J.T. George and E.M. Scott, Molecular clocks, type 2 diabetes and cardiovascular disease, Diab Vasc Dis Res 5 (2008), 89-95.

[55] J. Mendoza, P. Pevet and E. Challet, High-fat feeding alters the clock synchronization to light, J Physiol 586 (2008), 59015910.

[56] M. Garaulet and J.A. Madrid, Chronobiology, genetics and metabolic syndrome, Curr Opin Lipidol 20 (2009), 127-34.

[57] T. Akerstedt, Shift work and disturbed sleep/wakefulness, Sleep Med Rev 2 (1998), 117-128.

[58]A. A. van Mark, S.W. Weiler, M. Schröder, A. Otto, K Jauch-Chara, D.A. Groneberg, M. Spallek, R. Kessel and B. Kalsdorf, The impact of shift work induced chronic circadian disruption on IL-6 and TNF-alpha immune responses, J Occup Med Toxicol 5 (2010), 5- 18

[59] National Sleep Foundation: Sleep in America Poll, National Sleep Foundation, Washington, DC, 2005. Available from: www.sleepfoundation.org/

[60] S.L. Paim, M.L. Pires, L.R. Bittencourt, R.S. Silva, R.F. Santos, A.M. Esteves, A.T. Barreto, S. Tufik and M.T. de Mello, Sleep complaints and polysomnographic findings: a study of nuclear power plant shift workers, Chronobiol Int 25(2008),321-31.

[61]T. Young, M. Palta, J. Dempsey, J. Skatrud, S. Weber and S. Badr, The occurrence of sleep-disordered breathing among middle-aged adults, N Engl J Med 328 (1993), 1230-5

[62] J.A. Walsleben, R.G. Norman and R.D. Novak, Sleep habits of Long Island Rail Road commuters, Sleep 22 (1999), 728734.

[63]J. Vioque, A. Torres and J. Quiles, Time spent watching television, sleep duration and obesity in adults living in Valencia, Spain, Int J Obes Relat Metab Disord 24 (2000), 1683-8.

[64] S. Taheri, L. Lin, D. Austin, T. Young and E. Mignot, Short sleep duration is associated with reduced leptin, elevated ghrelin and increased body mass index, PLoS Med 1 (2004), 210-7.

[65]K. Spiegel, E. Tasali, P. Penev and E. Van Cauter, Brief communication: Sleep curtailment in healthy young men is associated with decreased leptin levels, elevated ghrelin levels, and increased hunger and appetite, Ann Intern Med 141 (2004), 846-50.

[66]K. Spiegel, K. Knutson, R. Leproult, E. Tasali and E. Van Cauter, Sleep loss: a novel risk factor for insulin resistance and type 2 diabetes, J Appl Physiol 99 (2005), 2008-2019.

[67]E.Van Cauter, J.D. Blackman, D. Roland, J.P. Spire, S. Refetoff and K.S. Polonsky, Modulation of glucose regulation and insulin secretion by circadian rhythmicity and sleep, J Clin Invest 88 (1991), 934-942.

[68]K. Spiegel, R. Leproult and E. Van Cauter, Impact of sleep debt on metabolic and endocrine function, Lancet 354 (1999), 1435-9.

[69] J.M. Mullington, J.L. Chan, H.P.Van Dongen, Sleep loss reduces diurnal rhythm amplitude of leptin in healthy men, $\mathrm{J}$ Neuroendocrinol 15 (2003), 851-4.

[70]B. Bodosi, J. Gardi, I. Hajdu, E. Szentirmai, F. Obal and J.M Krueger, Rhythms of ghrelin, leptin, and sleep in rats: effects of the normal diurnal cycle, restricted feeding, and sleep deprivation, Am J Physiol Regul Integr Comp Physiol 287 (2004), 1071-9.

[71]K. Spiegel, R. Leproult, M. L'hermite-Baleriaux, G. Copinschi, P.D. Penev and E. Van Cauter, Leptin levels are dependent on sleep duration: relationships with sympathovagal balance, carbohydrate regulation, cortisol, and thyrotropin, J Clin Endocrinol Metab 89 (2004), 5762-71.

[72] S. Taheri, The link between short sleep duration and obesity: we should recommend more sleep to prevent obesity, Arch Dis Child 91(2006), 881-4.

[73]C.A. Magee, X. Huang and D.C. Iverson, Acute sleep restriction alters neuroendocrine hormones and appetite in healthy male adults, Sleep Biol Rhythms 7 (2009), 125-127

[74]E. Van Cauter, K.S. Polonsky and A.J. Scheen, Roles of circadian rhythmicity and sleep in human glucose regulation, Endocrine Rev 18 (1997), 716-738.

[75]G. Hasler, D.J. Buysse, R. Klaghofer, A. Gamma, V. Ajdacic and D. Eich, The association between short sleep duration and obesity in young adults: a 13-year prospective study, Sleep 27 (2004),661-6

[76]D.F. Kripke, L. Garfinkel, D.L. Wingard, M.R. Klauber and M.R. Marler, Mortality associated with sleep duration and insomnia, Arch Gen Psychiatry 59 (2002), 131-6. 
[77]P. Naitoh, Sleep deprivation in human subjects: a reappraisal, Waking Sleeping 1 (1976), 53-60.

[78] N. Sudo and R. Ohtsuka, Nutrient intake among female shift workers in a computer factory in Japan, Int J Food Sci Nutr 52 (2001), 367-78.

[79] S. Armstrong, A chronometric approach to the study of feeding behaviour. Neurosci Biobehav Rev 4 (1980),27-53

[80] C.E.Adams and K.J. Morgan, Periodicity of eating: implications for human food consumption, Nutr Res 1 (1981), 525-50.

[81] W.P. Verboeket-van de Venne and K.R. Westerterp, Influence of the feeding frequency on nutrient utilization in man: consequences for energy metabolism, Eur J Clin Nutr 45 (1991), 161-9.

[82] J.G. Moore, Chronobiology of the gastrointestinal system, In: Y. Touitou and E. Haus. Biological rhythms in clinical and laboratory medicine, Berlin, Springer-Verlag, 1992, p. 410-7.

[83] A.V. Nedeltcheva, J.M. Kilkus, J. Imperial, D.A. Schoeller and P.D. Penev, Insufficient sleep undermines dietary efforts to reduce adiposity, Ann Intern Med 153 (2010),435-41.
[84]M. Dattilo, C.A. Crispim, I.Z. Zimberg, S. Tufik and M.T. de Mello, Meal distribution across the day and its relationship with body composition, Biol Rhythm Res 42 (2010), 119-129.

[85]J.M. De Castro, The time of day of food intake influences overall intake in humans, J Nutr 134 (2004), 104-11.

[86] N.K. Grupta, W.H. Mueller, W. Chan and J.C. Meininger, Is obesity associated with poor sleep quality in adolescents? Am J Hum Biol 14 (2002), 762-8.

[87]F.B. Ortega, P. Chillón and J.R. Ruiz, Sleep patterns in Spanish adolescents: associations with TV watching and leisuretime physical activity, Eur J Appl Physiol 110 (2010), 563-73.

[88] A.k. Spiegel, E. Tasali, R. Leproult and E. Van Cauter, Effects of poor and short sleep on glucose metabolism and obesity risk, Nat Rev Endocrinol 5 (2009), 253-61. 